\title{
Erratum to: Peritumoral apparent diffusion coefficients for prediction of lymphovascular invasion in clinically node-negative invasive breast cancer
}

\author{
Naoko Mori ${ }^{1}$ - Shunji Mugikura ${ }^{1} \cdot$ Chiaki Takasawa $^{1}$ - Minoru Miyashita ${ }^{2}$. \\ Akiko Shimauchi $^{1} \cdot$ Hideki Ota $^{1} \cdot$ Takanori Ishida $^{2} \cdot$ Atsuko Kasajima $^{3} \cdot$ Kei Takase $^{1}$. \\ Tetsuya Kodama ${ }^{4} \cdot$ Shoki Takahashi $^{1}$
}

Published online: 3 July 2015

(C) European Society of Radiology 2015

Erratum to: Eur Radiol

DOI 10.1007/s00330-015-3847-4

Due to a transcription error, Figure 1 was mistakenly included as Figure 2. Please find the correct Figure 2 below.

We apologise for this error.

The online version of the original article can be found at $\mathrm{http}: / / \mathrm{dx} . \mathrm{doi} . \mathrm{org} /$ 10.1007/s00330-015-3847-4.

\footnotetext{
Naoko Mori

naokomori7127@gmail.com
}

1 Department of Diagnostic Radiology, Tohoku University Graduate School of Medicine, Seiryo 1-1, Sendai 980-8574, Japan

2 Department of Surgical Oncology, Tohoku University Graduate School of Medicine, Seiryo 1-1, Sendai 980-8574, Japan

3 Department of Pathology, Tohoku University Graduate School of Medicine, Seiryo 1-1, Sendai 980-8574, Japan

4 Department of Biomedical Engineering, Tohoku University Graduate School of Medicine, Seiryo 1-1, Sendai 980-8574, Japan 

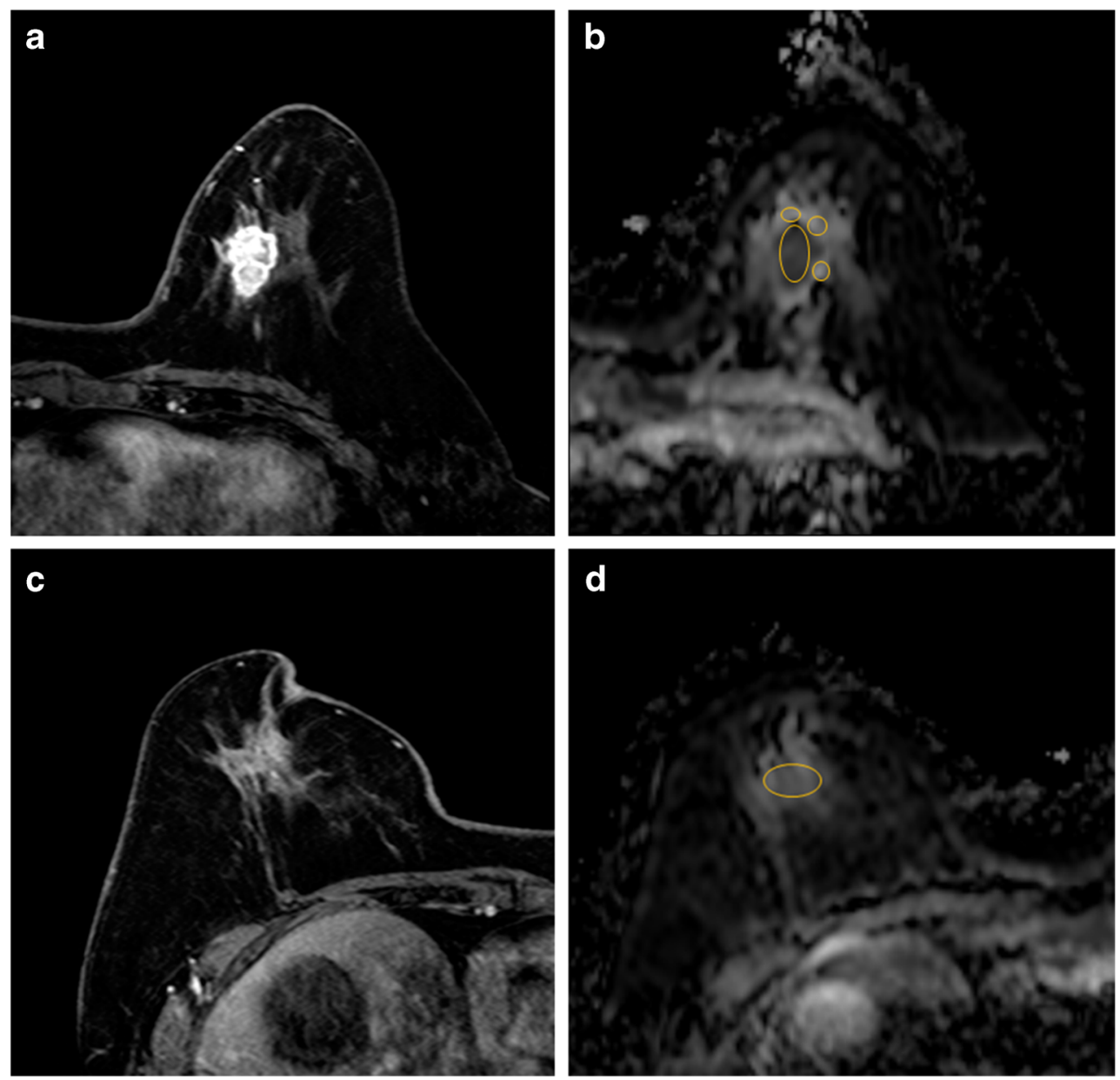

Fig. 2 Dynamic contrast-enhanced imaging (a, c) and ADC maps (b, d) in a 77-year-old woman with invasive breast cancer NOS diagnosed by biopsy to show the method used for placing ROIs to obtain the tumour$\mathrm{ADC}$, peritumoral maximum-ADC and the mean ADC of the contralateral breast parenchyma. Regarding the tumour-ADC, the slice with the largest tumour cross-section is selected and the largest oval or round ROI is placed inside the tumour (b) with reference to dynamic contrast-enhanced imaging (a). The mean value of the ROI (1047x $10 \mathrm{~mm} / \mathrm{s}$ ) is recorded as the tumour-ADC. For the peritumoral maximum-ADC, three ROIs are placed where the ADC visually appears to be most increased on breast parenchymal tissue adjacent to

the tumour contour on ADC maps (b): the three ROIs are 2054, 1892 and $1778 \times 10 \mathrm{~mm} / \mathrm{s}$, respectively. The maximum value $(2054 \times 10 \mathrm{~mm} / \mathrm{s})$ is recorded as the peritumoral maximum-ADC. On 3 slices of ADC maps, a ROI as large as possible is subsequently placed on the contralateral normal breast parenchyma (mean ADC values=1226, 1289 and $1481 \times$ $10 \mathrm{~mm} / \mathrm{s}$, respectively) not to include abnormally enhanced breast parenchyma or fat intervening the breast tissue (d) with reference to dynamic contrast-enhanced images (c). The mean value of the three ROIs $(1332 \times 10 \mathrm{~mm} / \mathrm{s})$ is recorded as the mean ADC of the contralateral breast parenchyma 